\title{
Modulating Applied Task Performance via Transcranial Electrical Stimulation
}

\author{
Tad T. Brunyé ${ }^{1,2,3 *}$, Erika K. Hussey 1,2, Eduardo B. Fontes ${ }^{3,4}$ and Nathan Ward ${ }^{3,4}$ \\ ${ }^{1}$ Center for Applied Brain and Cognitive Sciences, School of Engineering, Tufts University, Medford, MA, United States, \\ ${ }^{2}$ U.S. Army Combat Capabilities Development Command, Soldier Center (CCDC-SC), Natick, MA, United States, \\ ${ }^{3}$ Department of Psychology, Tufts University, Medford, MA, United States, ${ }^{4}$ NEUROEX-Research Group in Physical Activity, \\ Cognition and Behavior, Health Science Center, Department of Physical Education, Federal University of Rio Grande do \\ Norte, Natal, Brazil
}

Basic and applied research are increasingly adopting transcranial electrical stimulation (tES) for modulating perceptual, cognitive, affective, and motor processes. Industry and defense applications of tES hold potential for accelerating training and knowledge acquisition and sustaining work-related performance in the face of fatigue, workload, and stress. This mini-review article describes the promises and perils of tES, and reviews research testing its influence on two broad applied areas: sustaining and dividing attention, and operating in virtual environments. Also included is a discussion of challenges related to viable mechanistic explanations for tES effectiveness, attempts at replication and consideration of null results, and the potential importance of individual differences in predicting tES influences on human performance. Finally, future research directions are proposed to address these challenges and help develop a fuller understanding of tES viability for enhancing real-world performance.

\section{OPEN ACCESS}

Edited by:

Hasan Ayaz,

Drexel University, United States

Reviewed by:

Leila Chaieb,

University of Bonn, Germany

Abhishek Datta,

Soterix Medical, United States

${ }^{*}$ Correspondence:

Tad T. Brunyé

tbruny01@tufts.edu

Received: 17 January 2019 Accepted: 10 April 2019

Published: 30 April 2019

Citation:

Brunyé TT, Hussey EK, Fontes EB and Ward N (2019) Modulating Applied Task Performance via Transcranial Electrical Stimulation. Front. Hum. Neurosci. 13:140. doi: 10.3389/fnhum.2019.00140
Keywords: neuroergonomics, transcranial electrical stimulation, vigilance, multitasking, driving, navigation, virtual environments

\section{INTRODUCTION}

Transcranial electrical stimulation (tES) involves administering low intensity electrical current to superficial cortical regions by way of two or more electrodes mounted on the surface of the scalp (Nitsche et al., 2008; Silva et al., 2008; Woods et al., 2016). Electrical current propagates through the scalp, skull, and dura mater and into cortical tissue; experiments with animals and modeling efforts demonstrate that tES can produce subthreshold depolarization of cortical pyramidal and glial cells (Ruohonen and Karhu, 2012; Molaee-Ardekani et al., 2013; Rahman et al., 2013). This is only one of many putative explanations for tES effects on human performance, the mechanisms of which remain somewhat elusive (Bestmann et al., 2015). It is generally accepted that tES modulates neuronal and neurotransmitter activity, underlying its effects on perceptual, cognitive, affective and motor processes seen in clinical, rehabilitation, educational, and recreational contexts. There are several methods for administering tES in the laboratory, with multiple methodological parameters demonstrated to independently and/or interactively influence the specificity, directionality, robustness and reliability of tES effects on human performance (Paulus, 2011; Reed and Cohen Kadosh, 2018; Yavari et al., 2018). This is a large and complex parameter space that deserves considerable attention from the scientific communities and is an important hurdle to overcome before tES can be applied reliably, selectively, and safely in real-world contexts. 
This complexity has been compounded by a recent commercialization of tES for applications outside of the laboratory (Wurzman et al., 2016). It is important to note that most consumer-grade tES devices are not regulated by the United States Food and Drug Administration (FDA), are not considered low risk (FDA, 2016), and should be viewed with skepticism. Technological advances have, however, opened the door for neuroergonomics research to pursue tES research outside of the laboratory, which will benefit from the theories, tools, and techniques developed in the lab. Understanding the circumstances under which tES reliably alters human performance will help define and prioritize the real-world contexts and tasks that may prove suitable for tES application. The present review considers two broad areas that may prove fruitful for translational tES research: sustaining and dividing attention, and operating in virtual environments (Table 1).

\section{tES AND APPLIED TASKS}

Most tES research concerned with human performance focuses on modulating cognitive control processes by stimulating the dorsolateral prefrontal cortex (DLPFC; Brunoni and Vanderhasselt, 2014). The DLPFC supports a range of processes involved in controlling top-down control of complex voluntary actions, including solving complex tasks, inhibiting habitual responding, and correcting errors (MacDonald et al., 2000; Koechlin and Summerfield, 2007). Traditionally, DLPFC is targeted using bipolar electrode montages (i.e., one anode and one cathode). Complementing this work, researchers have begun to use multi-electrode montages arranged to target additional brain regions guided by finite element modeling, such as the hippocampus (Nikolin et al., 2015; Brunyé et al., 2018a), resting state motor network (Fischer et al., 2017), temporoparietal junction (Slaby et al., 2015), and inferior frontal cortex (Hussey et al., 2015; Hogeveen et al., 2016). Beyond adjusting the spatial properties of montages, recent work has also examined the temporal parameters of tES, including modifying dose (i.e., multiple days; longer administration times; Iyer et al., 2005; Reis et al., 2009; Hill et al., 2016), delivery time (i.e., online vs. offline stimulation; Pirulli et al., 2013), and frequency or phase properties (i.e., transcranial random noise stimulation, or tRNS; transcranial alternating current stimulation, or tACS; Filmer et al., 2014; Santarnecchi et al., 2015). This methodological variability is pervasive across basic and applied domains.

\section{Sustaining and Dividing Attention}

Sustained attention, or vigilance, involves maintaining alertness and focus to goal-related but infrequent stimuli over extended periods of time (Davies and Parasuraman, 1982). Example work-related tasks include airport luggage screening, air traffic control, military checkpoints and image analysis, industrial quality control, driving, and many medical tasks such as histology screening (Warm et al., 2008). In these cases, operators are trained to maintain attentiveness to ensure the detection of rare but valuable targets. Studies have shown that vigilance declines as the time on task increases, as reflected by a decrease in target detections and increase in reaction times (Mackworth, 1948; Helton et al., 2007; Warm and Parasuraman, 2009). Functional magnetic resonance imaging (fMRI) and positron emission tomography (PET) have consistently revealed that sustaining attention recruits a network of brain regions including lateral prefrontal cortices, parietal lobes, and the temporoparietal region (Pardo et al., 1991; Coull et al., 1998; Breckel et al., 2011), making vigilance tasks an ideal target for tES.

TABLE 1 | Overview of primary brain regions implicated in applied tasks, and existing research examining transcranial electrical stimulation (tES) effects on each applied task category.

\begin{tabular}{|c|c|c|}
\hline Applied task & Brain regions implicated & Existing tES research \\
\hline Sustained attention & $\begin{array}{l}\text { Lateral prefrontal cortices } \\
\text { Parietal lobes } \\
\text { Temporoparietal region }\end{array}$ & $\begin{array}{l}\text { Mclntire et al. (2014), Nelson et al. (2014), Mauri et al. (2015), } \\
\text { Kasten et al. (2016) and Loffler et al. (2018) }\end{array}$ \\
\hline Threat detection and identification & $\begin{array}{l}\text { Lateral PFC } \\
\text { Anterior cingulate cortex } \\
\text { Amygdala }\end{array}$ & Clark et al. (2012) and McKinley et al. (2013) \\
\hline Divided attention, multitasking & $\begin{array}{l}\text { Dorsolateral PFC } \\
\text { Intraparietal sulcus } \\
\text { Posterior lateral PFC } \\
\text { Cerebellum }\end{array}$ & $\begin{array}{l}\text { Filmer et al. (2013a,b), Nelson et al. (2016), Hsu et al. (2017) } \\
\text { and Hsu et al. (2018) }\end{array}$ \\
\hline Navigation and wayfinding & $\begin{array}{l}\text { Medial and right inferior parietal cortex } \\
\text { Posterior cingulate cortex } \\
\text { Left PFC } \\
\text { Medial temporal region } \\
\text { Parahippocampal gyrus } \\
\text { Hippocampus } \\
\text { Retrosplenial complex }\end{array}$ & Brunyé et al. (2014, 2018a) and Hampstead et al. (2014) \\
\hline Vehicle driving & $\begin{array}{l}\text { Dorsolateral PFC } \\
\text { Pre-supplementary motor area } \\
\text { Superior parietal cortex } \\
\text { Lateral occipital cortex } \\
\text { Cerebellum }\end{array}$ & Beeli et al. (2008a,b) and Sakai et al. (2014) \\
\hline
\end{tabular}


Several studies have examined the link between prefrontal tES and vigilance task performance. First, Nelson et al. (2014) examined whether tDCS targeting the left DLPFC with anodal stimulation (relative to cathodal stimulation over the same region) would influence performance on a simulated air traffic control task. Anodal stimulation caused an increase in hit rates and decrease in false alarm rates relative to the cathodal and sham conditions, and this effect persisted throughout the 40-min task. Thus, there was some evidence that anodal tDCS targeting the left DLPFC can reduce some of the typical vigilance decrements seen in hit and false alarm rates, but not reaction times. In a second study, McIntire et al. (2014) examined whether anodal tDCS over the left DLPFC (with an extra-cephalic cathode) would mitigate sleep-deprivation induced vigilance decrements. They found that tDCS (vs. sham) induced sustained performance on two vigilance tasks (a psychomotor vigilance task and the Mackworth clock task), in the form of accuracy and response times. Using tACS, studies are equivocal: one showed no reliable effect of alpha-band tACS targeting posterior brain areas on visual vigilance performance (Kasten et al., 2016), and another showed a reduced vigilance decrement with gamma-band tACS over the visual cortex (Loffler et al., 2018). Using tRNS, one study showed that tRNS reduced reaction times during sustained vigilance on a continuous performance task (Mauri et al., 2015).

Some vigilance tasks involve highly specialized knowledge, such as with medical imaging, airport luggage screening, and monitoring radar systems for emergent threats (Lesgold et al., 1988; Patel et al., 2005; Ericsson et al., 2006). Some research has assessed whether tES may hold value for accelerating the acquisition of knowledge and skills required to successfully perform threat detection and identification tasks (Parasuraman and McKinley, 2014). Clark et al. (2012) administered anodal tDCS to the right inferior frontal cortex or right parietal cortex, and assessed the rate and amount of learning in a concealed object learning task. They found that both tDCS montages produced robust and reliable increases in accuracy, reflecting accelerated learning of the vigilance and threat detection task. A second study found similar results with anodal tDCS targeting the ventrolateral PFC, with increased performance on a learning task involving threat detection (object recognition; McKinley et al., 2013). Thus, there is some evidence that anodal tDCS targeting the PFC can reduce the typical vigilance decrements seen with extended time on task, and accelerate the learning of critical cues that are important for detecting threats during vigilance tasks.

Just as sustaining attention underlies a wide range of outcomes, dividing attention, or multitasking is a common demand imposed by several real-world tasks. Decades of research have demonstrated considerable costs to accuracy and response times when operators attempt to multitask, and these decrements are typically attributed to central interference imposed by competing tasks and responses (sometimes referred to as a central bottleneck; Pashler, 1994; Marois and Ivanoff, 2005). The posterior lateral PFC (pLPFC) has been identified as playing a critical role in the stimulus-response mappings that underlie effective dual-tasking components of multitasking
(Jiang and Kanwisher, 2003; Dux et al., 2006, 2009). A few studies have examined whether tES targeting the left pLPFC would modulate multitasking performance, and results are equivocal. One study examined whether anodal or cathodal stimulation of the left pLPFC would modulate reaction times on simultaneous auditory and visual tasks (Filmer et al., 2013a). Cathodal tDCS reduced typical multitasking costs relative to anodal or sham stimulation, suggesting that cathodal stimulation may reduce neural noise and increase the signal to noise ratio in the pLPFC (Dockery et al., 2009; Miniussi et al., 2013). In a second study, the same research group demonstrated that anodal and cathodal tDCS targeting the left pLPFC reduced the typical performance advantages seen with multitasking training (Filmer et al., 2013b). Thus, there is evidence that cathodal pLPFC stimulation may improve multitasking performance, but that anodal or cathodal stimulation of the same region may interfere with multitasking training effectiveness. In both cases, the authors point to a critical causal role of this brain region for stimulus-response mapping, though the discrepant findings remain unresolved (Nikolin et al., 2019).

In a final study, Nelson et al. (2016) targeted the left DLPFC with anodal tDCS and found increased multitasking throughput capacity relative to sham stimulation. It is possible that a relatively domain-general increase in attentional control via DLPFC stimulation can induce enhancements on multitasking tasks, without specifically targeting the pLPFC (Strobach and Antonenko, 2017). This possibility is supported by studies demonstrating enhanced dual-tasking performance with tDCS targeting the inferior frontal (Strobach et al., 2015) and dorsolateral prefrontal cortices (Zhou et al., 2014). There is also evidence that tACS targeting theta oscillations in the PFC can induce multitasking improvements (Hsu et al., 2017, 2018). No research has directly examined the effects of tRNS on multitasking performance, though some suggest limited utility of tRNS for altering aspects of executive function reliant on the PFC (Mulquiney et al., 2011).

\section{Operating in Virtual Environments}

Virtual reality tools are becoming increasingly integrated into applied psychology paradigms to create immersive, lifelike scenarios that mimic the demands encountered outside of the lab. While combining neurostimulation and virtual reality holds potential for several applied domains (Teo et al., 2016), two specific examples are navigation and driving.

Navigating between waypoints in large-scale environments relies on a collection of cognitive processes, including spatial attention, perception, mental rotation, visualization, and working memory (Byrne et al., 2007). This diversity of processing demands is matched by a highly distributed network of brain regions (Vogt et al., 1992; Brotchie et al., 1995; Xu and Chun, 2006; Burgess, 2008; Harvey et al., 2012; Wiener et al., 2016; Boccia et al., 2017), each of which presents targeting opportunities for tES (Brunyé, 2018). In one study, researchers targeted the right medial temporal lobe with a multi-electrode tDCS montage and demonstrated no main effect 
of anodal stimulation on the ability to efficiently navigate a virtual environment (Brunyé et al., 2014); they did, however, find some evidence that it benefitted individuals with relatively low spatial sense of direction. In a second virtual navigation study, researchers used finite element modeling to inform electrode positions targeting the right hippocampus and parahippocampus with anodal tDCS, and found no significant influence on a virtual navigation task involving spatial encoding and memory retrieval (Brunyé et al., 2018a). Continuing research may benefit from leveraging functional connectivity between cortical and subcortical regions, targeting cortical targets to indirectly modulate brain activity in medial brain regions (Hampstead et al., 2014; Brunyé, 2018), and possibly extending research to tACS and tRNS.

Like spatial navigation, vehicle driving engages a multitude of perceptual and cognitive processes, resulting in effectively processing driving-related information and translating that into effective and safe control strategies. One brain region frequently implicated in this task is the right and left DLPFC: studies demonstrate activity in the right DLPFC in a vehicle-following task requiring the maintenance of a specific lead distance, left DLPFC activity in a rural driving simulation, and right DLPFC activity when attending to traffic rules during driving (Spiers and Maguire, 2007; Just et al., 2008; Uchiyama et al., 2012). Still others find no evidence of DLPFC activity during simulated or actual vehicle driving tasks (Calhoun et al., 2002; Horikawa et al., 2005; Jeong et al., 2006).

To our knowledge, only three published studies to date have examined tES influences on driver behavior, all using tDCS (no tACS or tRNS). In the first, Beeli et al. (2008a) administered anodal or cathodal tDCS over the left or right DLPFC during simulated driving, and found that both anodal stimulation conditions caused a reduction of risky driving behavior (increased following distance, reduced speed). Another study replicated these findings and demonstrated that speed violations and revolutions per minute were also reduced during a simulated driving scenario with anodal tDCS over left or right DLPFC relative to sham (Beeli et al., 2008b). Finally, Sakai et al. (2014) administered anodal or cathodal stimulation over the right or left DLPFC, and found that right anodal improved following distances and lane-keeping performance relative to sham or left anodal stimulation. Thus, there is some evidence that variations of DLPFC stimulation can enhance certain safety-related aspects of driving.

\section{tES CHALLENGES FOR APPLICATION}

We point to three primary challenges in considering tES for application to applied contexts and tasks. First, safe and effective tES administration relies on a highly complex and underspecified parameter space (Soekadar et al., 2016). These include variation in targeted brain regions, electrode positioning and sizing, stimulation intensity, timing and duration, and the polarity (anodal, cathodal) of tDCS and frequency of tACS. Furthermore, challenges associated with the state-dependence of the cortex and differential responsiveness to tES (Dockery et al., 2009; Bikson et al., 2013; Antal et al., 2014) may prove especially important for real-world applications. Specifically, the complexity of endogenous neural activations during real-world tasks may modulate tES effects on behavior in unknown ways. These parameters are complicated by the complexity of current propagation through tissue and varied neuronal morphology, the non-linear excitability gradients of tES, varied device quality and reliability, and the potential for participants to detect differences between active and sham conditions. These challenges warrant caution among practitioners who seek to apply tES in contexts outside of the laboratory where they may realize reduced control over these factors. This is especially relevant given the heightened interest in extending applied cognitive tasks to immersive, ambulatory virtual environments, particularly in rehabilitation contexts (Rothwell, 2012; Viana et al., 2014; Massetti et al., 2017).

Second, recent research has suggested that individual differences in brain morphology, knowledge, skills and abilities play a role in predicting tES impacts. As noted previously, spatial skills predicted whether tDCS influenced navigation performance (Brunyé et al., 2014); it could be the case that those with high spatial skills have relatively optimized neural network dynamics during complex spatial tasks, and tES can interfere with those relatively refined activity patterns. Similar influences of individual differences in predicting tES influences have been found in several recent studies: high creative potential is linked to increased breadth of semantic associations with left frontopolar tDCS (Brunyé et al., 2015), working memory capacity and educational level are linked to increased neural activity and cognitive function with left DLPFC tDCS (Berryhill and Jones, 2012; Jones et al., 2015), and trait mathematics anxiety is linked to whether left DLPFC tDCS improves arithmetic task performance (Sarkar et al., 2014). Continuing research will likely find additional predictive value in trait-based measures, and the field will continue to benefit from neuroimaging in at least three ways: individualized targeting of brain regions (Bikson et al., 2012), closed-loop stimulation triggering using structural and functional brain imaging (McKendrick et al., 2015), and improved mechanistic understandings of tES effects at the level of neurons, neural networks, and behavior (Filmer et al., 2014; Soekadar et al., 2016).

Third, at least partially due to the complex parameter space of tES and individual variability in the robustness and directionality of behavioral responses, it is important to consider studies demonstrating null and negative effects of tES. Recent meta-analyses are equivocal in determining whether tES induce reliable effects on perceptual and cognitive processes in healthy adults (Jacobson et al., 2012; Chhatbar and Feng, 2015; Horvath et al., 2015a,b). Furthermore, some studies have found unexpected negative results of tDCS targeting the DLPFC, suggesting that tES is not a one-size-fits-all solution for all individuals, contexts, and tasks (Zwissler et al., 2014; Crivelli and Balconi, 2017; Brunyé et al., 2018b). Moving forward, we argue for the use of more rigorous and reproducible methods in tES research, especially when it comes to less standardized applied tasks and outcome measures. This may 
include establishing required sample sizes, procedures, predicted effect sizes, and analysis approaches in advance (e.g., through preregistered reports). Furthermore, while tDCS is increasingly used in relatively applied contexts, research using tACS and tRNS in applied domains is largely limited to areas such as motor learning (Nitsche et al., 2003) and procedural skill acquisition (Tecchio et al., 2010).

\section{CONCLUSION}

tES may prove valuable for modulating applied task performance, though research in this area warrants careful consideration of several individual-, context-, and task-related factors that may predict the robustness and directionality of tES effects. Whereas most applied research with tES has administered tDCS, tACS and tRNS have also shown potential to modulate cortical activity and behavior. Even in highly applied and dynamic tasks, such as navigation and driving, tES appears to carry some

\section{REFERENCES}

Antal, A., Ambrus, G. G., and Chaieb, L. (2014). Toward unraveling readingrelated modulations of tdcs-induced neuroplasticity in the human visual cortex. Front. Psychol. 5:642. doi: 10.3389/fpsyg.2014.00642

Beeli, G., Casutt, G., Baumgartner, T., and Jäncke, L. (2008a). Modulating presence and impulsiveness by external stimulation of the brain. Behav. Brain Funct. 4:33. doi: 10.1186/1744-9081-4-33

Beeli, G., Koeneke, S., Gasser, K., and Jäncke, L. (2008b). Brain stimulation modulates driving behavior. Behav. Brain Funct. 4:34. doi: 10.1186/17449081-4-34

Berryhill, M. E., and Jones, K. T. (2012). tDCS selectively improves working memory in older adults with more education. Neurosci. Lett. 521, 148-151. doi: 10.1016/j.neulet.2012.05.074

Bestmann, S., de Berker, A. O., and Bonaiuto, J. (2015). Understanding the behavioural consequences of noninvasive brain stimulation. Trends Cogn. Sci. 19, 13-20. doi: 10.1016/j.tics.2014.10.003

Bikson, M., Name, A., and Rahman, A. (2013). Origins of specificity during tDCS: anatomical, activity-selective, and input-bias mechanisms. Front. Hum. Neurosci. 7:688. doi: 10.3389/fnhum.2013.00688

Bikson, M., Rahman, A., Datta, A., Fregni, F., and Merabet, L. (2012). High-resolution modeling assisted design of customized and individualized transcranial direct current stimulation protocols. Neuromodulation 15, 306-315. doi: 10.1111/j.1525-1403.2012.00481.x

Boccia, M., Sulpizio, V., Nemmi, F., Guariglia, C., and Galati, G. (2017). Direct and indirect parieto-medial temporal pathways for spatial navigation in humans: evidence from resting-state functional connectivity. Brain Struct. Funct. 222, 1945-1957. doi: 10.1007/s00429-016-1318-6

Breckel, T. P. K., Giessing, C., and Thiel, C. M. (2011). Impact of brain networks involved in vigilance on processing irrelevant visual motion. Neuroimage 55, 1754-1762. doi: 10.1016/j.neuroimage.2011.01.025

Brotchie, P. R., Andersen, R. A., Snyder, L. H., and Goodman, S. J. (1995). Head position signals used by parietal neurons to encode locations of visual stimuli. Nature 375, 232-235. doi: 10.1038/375232a0

Brunoni, A. R., and Vanderhasselt, M.-A. (2014). Working memory improvement with non-invasive brain stimulation of the dorsolateral prefrontal cortex: a systematic review and meta-analysis. Brain Cogn. 86, 1-9. doi: 10.1016/j.bandc. 2014.01.008

Brunyé, T. T. (2018). Modulating spatial processes and navigation via transcranial electrical stimulation: a mini review. Front. Hum. Neurosci. 11:649. doi: 10.3389/fnhum.2017.00649

Brunyé, T. T., Holmes, A., Cantelon, J., Eddy, M. D., Gardony, A. L., Mahoney, C. R., et al. (2014). Direct current brain stimulation enhances navigation efficiency in individuals with low sense of direction. Neuroreport 25, 1175-1179. doi: 10.1097/wnr.0000000000000214 performance benefits. This is compelling because as tES is slowly incorporated into highly complex real-world environments and tasks, there is potential that its robustness and reliability may diminish relative to results found in controlled laboratory and simulation environments. Continuing research will benefit from transitioning tES out of the laboratory and simulation environment and examining such a possibility.

\section{AUTHOR CONTRIBUTIONS}

TB conceived the review and prepared the manuscript, with critical feedback and revisions from EH, EF, and NW.

\section{FUNDING}

This review was supported by applied research funding from the U.S. Army Combat Capabilities Development Command, Soldier Center (CCDC-SC; \#18-109).

Brunyé, T. T., Hussey, E. K., Gardony, A. L., Holmes, A., and Taylor, H. A. (2018a). Targeted right medial temporal lobe tDCS and associative spatial and non-spatial memory. J. Cogn. Enhanc. 3, 287-297. doi: 10.1007/s41465-018$0072-5$

Brunyé, T. T., Smith, A. M., Horner, C. B., and Thomas, A. K. (2018b). Verbal long-term memory is enhanced by retrieval practice but impaired by prefrontal direct current stimulation. Brain Cogn. 128, 80-88. doi: 10.1016/j.bandc.2018. 09.008

Brunyé, T. T., Moran, J. M., Cantelon, J., Holmes, A., Eddy, M. D., Mahoney, C. R., et al. (2015). Increasing breadth of semantic associations with left frontopolar direct current brain stimulation: a role for individual differences. Neuroreport 26, 296-301. doi: 10.1097/wnr.0000000000000348

Burgess, N. (2008). Spatial cognition and the brain. Ann. N Y Acad. Sci. 1124, 77-97. doi: 10.1196/annals.1440.002

Byrne, P., Becker, S., and Burgess, N. (2007). Remembering the past and imagining the future: a neural model of spatial memory and imagery. Psychol. Rev. 114, 340-375. doi: 10.1037/0033-295x.114.2.340

Calhoun, V. D., Pekar, J. J., McGinty, V. B., Adali, T., Watson, T. D., and Pearlson, G. D. (2002). Different activation dynamics in multiple neural systems during simulated driving. Hum. Brain Mapp. 16, 158-167. doi: $10.1002 / \mathrm{hbm} .10066$

Chhatbar, P. Y., and Feng, W. (2015). Data synthesis in meta-analysis may conclude differently on cognitive effect from transcranial direct current stimulation. Brain Stimul. 8, 974-976. doi: 10.1016/j.brs.2015. 06.001

Clark, V. P., Coffman, B. A., Mayer, A. R., Weisend, M. P., Lane, T. D. R., Calhoun, V. D., et al. (2012). TDCS guided using fMRI significantly accelerates learning to identify concealed objects. Neuroimage 59, 117-128. doi: 10.1016/j. neuroimage.2010.11.036

Coull, J. T., Frackowiak, R. S. J., and Frith, C. D. (1998). Monitoring for target objects: activation of right frontal and parietal cortices with increasing time on task. Neuropsychologia 36, 1325-1334. doi: 10.1016/s0028-3932(98) 00035-9

Crivelli, D., and Balconi, M. (2017). The agent brain: a review of non-invasive brain stimulation studies on sensing agency. Front. Behav. Neurosci. 11:229. doi: 10.3389/fnbeh.2017.00229

Davies, D. R., and Parasuraman, R. (1982). The Psychology of Vigilance. London: Academic Press.

Dockery, C. A., Hueckel-Weng, R., Birbaumer, N., and Plewnia, C. (2009). Enhancement of planning ability by transcranial direct current stimulation. J. Neurosci. 29, 7271-7277. doi: 10.1523/JNEUROSCI.006509.2009

Dux, P. E., Ivanoff, J., Asplund, C. L., and Marois, R. (2006). Isolation of a central bottleneck of information processing with time-resolved fMRI. Neuron 52, 1109-1120. doi: 10.1016/j.neuron.2006.11.009 
Dux, P. E., Tombu, M. N., Harrison, S., Rogers, B. P., Tong, F., and Marois, R. (2009). Training improves multitasking performance by increasing the speed of information processing in human prefrontal cortex. Neuron 63, 127-138. doi: 10.1016/j.neuron.2009.06.005

Ericsson, A. K., Charness, N., Feltovitch, P. J., and Hoffman, R. R. (2006). The Cambridge Handbook of Expertise and Expert Performance. Cambridge: Cambridge University Press.

FDA. (2016). General Wellness: Policy for Low Risk Devices Draft Guidance for Industry and Food and Drug Administration Staff. Silver Spring, MD: U.S. Department of Health and Human Services Food. Available online at: https://www.fda.gov/downloads/medicaldevices/ deviceregulationandguidance/guidancedocuments/ucm429674.pdf [Accessed March 25, 2019].

Filmer, H. L., Dux, P. E., and Mattingley, J. B. (2014). Applications of transcranial direct current stimulation for understanding brain function. Trends Neurosci. 37, 742-753. doi: 10.1016/j.tins.2014.08.003

Filmer, H. L., Mattingley, J. B., and Dux, P. E. (2013a). Improved multitasking following prefrontal tDCS. Cortex 49, 2845-2852. doi: 10.1016/j.cortex.2013. 08.015

Filmer, H. L., Mattingley, J. B., Marois, R., and Dux, P. E. (2013b). Disrupting prefrontal cortex prevents performance gains from sensory-motor training. J. Neurosci. 33, 18654-18660. doi: 10.1523/JNEUROSCI.2019-13.2013

Fischer, D. B., Fried, P. J., Ruffini, G., Ripolles, O., Salvador, R., Banus, J., et al. (2017). Multifocal tDCS targeting the resting state motor network increases cortical excitability beyond traditional tDCS targeting unilateral motor cortex. Neuroimage 157, 34-44. doi: 10.1016/j.neuroimage.2017.05.060

Hampstead, B. M., Brown, G. S., and Hartley, J. F. (2014). Transcranial direct current stimulation modulates activation and effective connectivity during spatial navigation. Brain Stimul. 7, 314-324. doi: 10.1016/j.brs.2013.12.006

Harvey, C. D., Coen, P., and Tank, D. W. (2012). Choice-specific sequences in parietal cortex during a virtual-navigation decision task. Nature 484, 62-68. doi: 10.1038/nature10918

Helton, W. S., Hollander, T. D., Warm, J. S., Tripp, L. D., Parsons, K., Matthews, G., et al. (2007). The abbreviated vigilance task and cerebral hemodynamics. J. Clin. Exp. Neuropsychol. 29, 545-552. doi: 10.1080/13803390600814757

Hill, A. T., Fitzgerald, P. B., and Hoy, K. E. (2016). Effects of anodal transcranial direct current stimulation on working memory: a systematic review and meta-analysis of findings from healthy and neuropsychiatric populations. Brain Stimul. 9, 197-208. doi: 10.1016/j.brs.2015.10.006

Hogeveen, J., Grafman, J., Aboseria, M., David, A., Bikson, M., and Hauner, K. K. (2016). Effects of high-definition and conventional tDCS on response inhibition. Brain Stimul. 9, 720-729. doi: 10.1016/j.brs.2016.04.015

Horikawa, E., Okamura, N., Tashiro, M., Sakurada, Y., Maruyama, M., Arai, H., et al. (2005). The neural correlates of driving performance identified using positron emission tomography. Brain Cogn. 58, 166-171. doi: 10.1016/j.bandc. 2004.10.002

Horvath, J. C., Forte, J. D., and Carter, O. (2015a). Evidence that transcranial direct current stimulation (tDCS) generates little-to-no reliable neurophysiologic effect beyond MEP amplitude modulation in healthy human subjects: a systematic review. Neuropsychologia 66, 213-236. doi: 10.1016/j. neuropsychologia.2014.11.021

Horvath, J. C., Forte, J. D., and Carter, O. (2015b). Quantitative review finds no evidence of cognitive effects in healthy populations from single-session transcranial direct current stimulation (tDCS). Brain Stimul. 8, 535-550. doi: 10.1016/j.brs.2015.01.400

Hsu, W. Y., Zanto, T. P., and Gazzaley, A. (2018). Parametric effects of transcranial alternating current stimulation on multitasking performance. Brain Stimul. 12, 73-83. doi: 10.1016/j.brs.2018.10.010

Hsu, W. Y., Zanto, T. P., van Schouwenburg, M. R., and Gazzaley, A. (2017). Enhancement of multitasking performance and neural oscillations by transcranial alternating current stimulation. PLoS One 12:e178579. doi: 10.1371/journal.pone.0178579

Hussey, E. K., Ward, N., Christianson, K., and Kramer, A. F. (2015). Language and memory improvements following tDCS of left lateral prefrontal cortex. PLoS One 10:e0141417. doi: 10.1371/journal.pone.0141417

Iyer, M. B., Mattu, U., Grafman, J., Lomarev, M., Sato, S., and Wassermann, E. M. (2005). Safety and cognitive effect of frontal DC brain polarization in healthy individuals. Neurology 64, 872-875. doi: 10.1212/01.wnl.0000152986. 07469.e9

Jacobson, L., Koslowsky, M., and Lavidor, M. (2012). TDCS polarity effects in motor and cognitive domains: a meta-analytical review. Exp. Brain Res. 216, 1-10. doi: 10.1007/s00221-011-2891-9

Jeong, M., Tashiro, M., Singh, L. N., Yamaguchi, K., Horikawa, E., Miyake, M., et al. (2006). Functional brain mapping of actual car-driving using $\left[{ }^{18}\right.$ F]FDG- PET. Ann. Nucl. Med. 20, 623-628. doi: 10.1007/bf029 84660

Jiang, Y., and Kanwisher, N. (2003). Common neural substrates for response selection across modalities and mapping paradigms. J. Cogn. Neurosci. 15, 1080-1094. doi: 10.1162/089892903322598067

Jones, K. T., Gözenman, F., and Berryhill, M. E. (2015). The strategy and motivational influences on the beneficial effect of neurostimulation: a tDCS and fNIRS study. Neuroimage 105, 238-247. doi: 10.1016/j.neuroimage.2014. 11.012

Just, M. A., Keller, T. A., and Cynkar, J. (2008). A decrease in brain activation associated with driving when listening to someone speak. Brain Res. 1205, 70-80. doi: 10.1016/j.brainres.2007.12.075

Kasten, F. H., Dowsett, J., and Herrmann, C. S. (2016). Sustained aftereffect of $\alpha$-tACS lasts up to $70 \mathrm{~min}$ after stimulation. Front. Hum. Neurosci. 10:245. doi: 10.3389/fnhum.2016.00245

Koechlin, E., and Summerfield, C. (2007). An information theoretical approach to prefrontal executive function. Trends Cogn. Sci. 11, 229-235. doi: 10.1016/j.tics. 2007.04.005

Lesgold, A., Rubinson, H., Feltovich, P., Glaser, R., Klopfer, D., and Wang, Y. (1988). "Expertise in a complex skill: diagnosing x-ray pictures," in The Nature of Expertise, eds M. T. H. Chi, R. Glaser and M. J. Farr (Hillsdale, NJ: Lawrence Erlbaum Associates), 311-342.

Loffler, B. S., Stecher, H. I., Fudickar, S., de Sordi, D., Otto-Sobotka, F., Hein, A., et al. (2018). Counteracting the slowdown of reaction times in a vigilance experiment with $40-\mathrm{Hz}$ transcranial alternating current stimulation. IEEE Trans. Neural Syst. Rehabil. Eng. 26, 2053-2061. doi: 10.1109/tnsre.2018. 2869471

MacDonald, A. W. III., Cohen, J. D., Stenger, V. A., and Carter, C. S. (2000). Dissociating the role of the dorsolateral prefrontal and anterior cingulate cortex in cognitive control. Science 288, 1835-1838. doi: 10.1126/science.288. 5472.1835

Mackworth, N. H. (1948). The breakdown of vigilance during prolonged visual search. Q. J. Exp. Psychol. 1, 6-21. doi: 10.1080/174702148084 16738

Marois, R., and Ivanoff, J. (2005). Capacity limits of information processing in the brain. Trends Cogn. Sci. 9, 296-305. doi: 10.1016/j.tics.2005. 04.010

Massetti, T., Crocetta, T. B., Silva, T. D. D., Trevizan, I. L., Arab, C., Caromano, F. A., et al. (2017). Application and outcomes of therapy combining transcranial direct current stimulation and virtual reality: a systematic review. Disabil. Rehabil. Assist. Technol. 12, 551-559. doi: 10.1080/17483107.2016. 1230152

Mauri, P., Miniussi, C., Balconi, M., and Brignani, D. (2015). Bursts of transcranial electrical stimulation increase arousal in a continuous performance test. Neuropsychologia 74, 127-136. doi: 10.1016/j.neuropsychologia.2015. 03.006

McIntire, L. K., McKinley, R. A., Goodyear, C., and Nelson, J. M. (2014). A comparison of the effects of transcranial direct current stimulation and caffeine on vigilance and cognitive performance during extended wakefulness. Brain Stimul. 7, 499-507. doi: 10.1016/j.brs.2014.04.008

McKendrick, R., Parasuraman, R., and Ayaz, H. (2015). Wearable functional near infrared spectroscopy (fNIRS) and transcranial direct current stimulation (tDCS): expanding vistas for neurocognitive augmentation. Front. Syst. Neurosci. 9:27. doi: 10.3389/fnsys.2015.00027

McKinley, R. A., McIntire, L., Bridges, N., Goodyear, C., Bangera, N. B., and Weisend, M. P. (2013). Acceleration of image analyst training with transcranial direct current stimulation. Behav. Neurosci. 127, 936-946. doi: $10.1037 / \mathrm{a} 0034975$

Miniussi, C., Harris, J. A., and Ruzzoli, M. (2013). Modelling non-invasive brain stimulation in cognitive neuroscience. Neurosci. Biobehav. Rev. 37, 1702-1712. doi: 10.1016/j.neubiorev.2013.06.014 
Molaee-Ardekani, B., Márquez-Ruiz, J., Merlet, I., Leal-Campanario, R., Gruart, A., Sánchez-Campusano, R., et al. (2013). Effects of transcranial Direct Current Stimulation (tDCS) on cortical activity: a computational modeling study. Brain Stimul. 6, 25-39. doi: 10.1016/j.brs.2011. 12.006

Mulquiney, P. G., Hoy, K. E., Daskalakis, Z. J., and Fitzgerald, P. B. (2011). Improving working memory: exploring the effect of transcranial random noise stimulation and transcranial direct current stimulation on the dorsolateral prefrontal cortex. Clin. Neurophysiol. 122, 2384-2389. doi: 10.1016/j.clinph. 2011.05.009

Nelson, J. T., McKinley, R. A., Golob, E. J., Warm, J. S., and Parasuraman, R. (2014). Enhancing vigilance in operators with prefrontal cortex transcranial direct current stimulation (tDCS). Neuroimage 85, 909-917. doi: 10.1016/j. neuroimage.2012.11.061

Nelson, J. T., McKinley, R. A., Phillips, C., McIntire, L. K., Goodyear, C., Kreiner, A., et al. (2016). The effects of transcranial direct current stimulation (tDCS) on multitasking throughput capacity. Front. Hum. Neurosci. 10:589. doi: 10.3389/fnhum.2016.00589

Nikolin, S., Lauf, S., Loo, C. K., and Martin, D. (2019). Effects of high-definition transcranial direct current stimulation (HD-tDCS) of the intraparietal sulcus and dorsolateral prefrontal cortex on working memory and divided attention. Front. Integr. Neurosci. 12:64. doi: 10.3389/fnint.2018. 00064

Nikolin, S., Loo, C. K., Bai, S., Dokos, S., and Martin, D. M. (2015). Focalised stimulation using high definition transcranial direct current stimulation (HD-tDCS) to investigate declarative verbal learning and memory functioning. Neuroimage 117, 11-19. doi: 10.1016/j.neuroimage.2015. 05.019

Nitsche, M. A., Cohen, L. G., Wassermann, E. M., Priori, A., Lang, N., Antal, A., et al. (2008). Transcranial direct current stimulation: state of the art 2008. Brain Stimul. 1, 206-223. doi: 10.1016/j.brs.2008.06.004

Nitsche, M. A., Schauenburg, A., Lang, N., Liebetanz, D., Exner, C., Paulus, W., et al. (2003). Facilitation of implicit motor learning by weak transcranial direct current stimulation of the primary motor cortex in the human. J. Cogn. Neurosci. 15, 619-626. doi: 10.1162/089892903321662994

Parasuraman, R., and McKinley, R. A. (2014). Using noninvasive brain stimulation to accelerate learning and enhance human performance. Hum. Factors 56, 816-824. doi: 10.1177/0018720814538815

Pardo, J. V., Fox, P. T., and Raichle, M. E. (1991). Localization of a human system for sustained attention by positron emission tomography. Nature 349, 61-64. doi: $10.1038 / 349061 \mathrm{a} 0$

Pashler, H. (1994). Dual-task interference in simple tasks: data and theory. Psychol. Bull. 116, 220-244. doi: 10.1037//0033-2909.116.2.220

Patel, V. L., Arocha, J., and Zhang, J. (2005). "Thinking and reasoning in medicine," in The Cambridge Handbook of Thinking and Reasoning, eds K. J. Holyoak and R. G. Morrison (Cambridge, UK: Cambridge University Press), 727-750.

Paulus, W. (2011). Transcranial electrical stimulation (tES-tDCS; tRNS, tACS) methods. Neuropsychol. Rehabil. 21, 602-617. doi: 10.1080/09602011.2011. 557292

Pirulli, C., Fertonani, A., and Miniussi, C. (2013). The role of timing in the induction of neuromodulation in perceptual learning by transcranial electric stimulation. Brain Stimul. 6, 683-689. doi: 10.1016/j.brs.2012. 12.005

Rahman, A., Reato, D., Arlotti, M., Gasca, F., Datta, A., Parra, L. C., et al. (2013). Cellular effects of acute direct current stimulation: somatic and synaptic terminal effects. J. Physiol. 591, 2563-2578. doi: 10.1113/jphysiol.2012. 247171

Reed, T., and Cohen Kadosh, R. (2018). Transcranial electrical stimulation (tES) mechanisms and its effects on cortical excitability and connectivity. J. Inherit. Metab. Dis. 41, 1123-1130. doi: 10.1007/s10545-018-0181-4

Reis, J., Schambra, H. M., Cohen, L. G., Buch, E. R., Fritsch, B., Zarahn, E., et al. (2009). Noninvasive cortical stimulation enhances motor skill acquisition over multiple days through an effect on consolidation. Proc. Natl. Acad. Sci. U S A 106, 1590-1595. doi: 10.1073/pnas.0805413106

Rothwell, J. C. (2012). Clinical applications of noninvasive electrical stimulation: problems and potential. Clin. EEG Neurosci. 43, 209-214. doi: $10.1177 / 1550059412444973$
Ruohonen, J., and Karhu, J. (2012). TDCS possibly stimulates glial cells. Clin. Neurophysiol. 123, 2006-2009. doi: 10.1016/j.clinph.2012. 02.082

Sakai, H., Uchiyama, Y., Tanaka, S., Sugawara, S. K., and Sadato, N. (2014). Prefrontal transcranial direct current stimulation improves fundamental vehicle control abilities. Behav. Brain Res. 273, 57-62. doi: 10.1016/j.bbr.2014. 07.036

Santarnecchi, E., Brem, A. K., Levenbaum, E., Thompson, T., Kadosh, R. C., and Pascual-Leone, A. (2015). Enhancing cognition using transcranial electrical stimulation. Curr. Opin. Behav. Sci. 4, 171-178. doi: 10.1016/j.cobeha.2015. 06.003

Sarkar, A., Dowker, A., and Cohen Kadosh, R. (2014). Cognitive enhancement or cognitive cost: trait-specific outcomes of brain stimulation in the case of mathematics anxiety. J. Neurosci. 34, 16605-16610. doi: 10.1523/JNEUROSCI. 3129-14.2014

Silva, S., Basser, P. J., and Miranda, P. C. (2008). Elucidating the mechanisms and loci of neuronal excitation by transcranial magnetic stimulation using a finite element model of a cortical sulcus. Clin. Neurophysiol. 119, 2405-2413. doi: 10.1016/j.clinph.2008.07.248

Slaby, I., Holmes, A., Moran, J. M., Eddy, M. D., Mahoney, C. R., Taylor, H. A., et al. (2015). Direct current stimulation of the left temporoparietal junction modulates dynamic humor appreciation. Neuroreport 26, 988-993. doi: $10.1097 /$ wnr. 0000000000000456

Soekadar, S. R., Herring, J. D., and McGonigle, D. (2016). Transcranial electric stimulation (tES) and NeuroImaging: the state-of-the-art, new insights and prospects in basic and clinical neuroscience. Neuroimage 140, 1-3. doi: 10.1016/j.neuroimage.2016.08.020

Spiers, H. J., and Maguire, E. A. (2007). Neural substrates of driving behaviour. Neuroimage 36, 245-255. doi: 10.1016/j.neuroimage.2007. 02.032

Strobach, T., and Antonenko, D. (2017). tDCS-induced effects on executive functioning and their cognitive mechanisms: a review. J. Cogn. Enhanc. 1, 49-64. doi: 10.1007/s41465-016-0004-1

Strobach, T., Soutschek, A., Antonenko, D., Flöel, A., and Schubert, T. (2015). Modulation of executive control in dual tasks with transcranial direct current stimulation (tDCS). Neuropsychologia 68, 8-20. doi: 10.1016/j. neuropsychologia.2014.12.024

Tecchio, F., Zappasodi, F., Assenza, G., Tombini, M., Vollaro, S., Barbati, G., et al. (2010). Anodal transcranial direct current stimulation enhances procedural consolidation. J. Neurophysiol. 104, 1134-1140. doi: 10.1152/jn.00 661.2009

Teo, W. P., Muthalib, M., Yamin, S., Hendy, A. M., Bramstedt, K., Kotsopoulos, E., et al. (2016). Does a combination of virtual reality, neuromodulation and neuroimaging provide a comprehensive platform for neurorehabilitation? - a narrative review of the literature. Front. Hum. Neurosci. 10:284. doi: 10.3389/fnhum.2016.00284

Uchiyama, Y., Toyoda, H., Sakai, H., Shin, D., Ebe, K., and Sadato, N. (2012). Suppression of brain activity related to a car-following task with an auditory task: an fMRI study. Trans. Res. F Traffic Psychol. Behav. 15, 25-37. doi: $10.1016 /$ j.trf.2011.11.002

Viana, R. T., Laurentino, G. E. C., Souza, R. J. P., Fonseca, J. B., Silva Filho, E. M., Dias, S. N., et al. (2014). Effects of the addition of transcranial direct current stimulation to virtual reality therapy after stroke: a pilot randomized controlled trial. NeuroRehabilitation 34, 437-446. doi: 10.3233/NRE141065

Vogt, B. A., Finch, D. M., and Olson, C. R. (1992). Functional heterogeneity in cingulate cortex: the anterior executive and posterior evaluative regions. Cereb. Cortex 2, 435-443. doi: 10.1093/cercor/2.6.435-a

Warm, J. S., and Parasuraman, R. (2009). "Cerebral hemodynamics and vigilance," in Neuroergonomics: The Brain at Work, eds R. Parasuraman and M. Rizzo (Oxford: Oxford University Press), 146-158.

Warm, J. S., Parasuraman, R., and Matthews, G. (2008). Vigilance requires hard mental work and is stressful. Hum. Factors 50, 433-441. doi: $10.1518 / 001872008 \times 312152$

Wiener, M., Michaelis, K., and Thompson, J. C. (2016). Functional correlates of likelihood and prior representations in a virtual distance task. Hum. Brain Mapp. 37, 3172-3187. doi: 10.1002/hbm. 23232 
Woods, A. J., Antal, A., Bikson, M., Boggio, P. S., Brunoni, A. R., Celnik, P., et al. (2016). A technical guide to tDCS, and related non-invasive brain stimulation tools. Clin. Neurophysiol. 127, 1031-1048. doi: 10.1016/j.clinph.2015.11.012

Wurzman, R., Hamilton, R. H., Pascual-Leone, A., and Fox, M. D. (2016). An open letter concerning do-it-yourself users of transcranial direct current stimulation. Ann. Neurol. 80, 1-4. doi: 10.1002/ana.24689

$\mathrm{Xu}, \mathrm{Y}$., and Chun, M. M. (2006). Dissociable neural mechanisms supporting visual short-term memory for objects. Nature 440, 91-95. doi: 10.1038/nature04262

Yavari, F., Jamil, A., Mosayebi Samani, M., Vidor, L. P., and Nitsche, M. A. (2018). Basic and functional effects of transcranial electrical stimulation (tES) - an introduction. Neurosci. Biobehav. Rev. 85, 81-92. doi: 10.1016/j.neubiorev. 2017.06.015

Zhou, J., Hao, Y., Wang, Y., Jor'dan, A., Pascual-Leone, A., Zhang, J., et al. (2014). Transcranial direct current stimulation reduces the cost of performing a cognitive task on gait and postural control. Eur. J. Neurosci. 39, 1343-1348. doi: 10.1111/ejn.12492
Zwissler, B., Sperber, C., Aigeldinger, S., Schindler, S., Kissler, J., and Plewnia, C. (2014). Shaping memory accuracy by left prefrontal transcranial direct current stimulation. J. Neurosci. 34, 4022-4026. doi: 10.1523/JNEUROSCI. 5407-13.2014

Conflict of Interest Statement: The authors declare that the research was conducted in the absence of any commercial or financial relationships that could be construed as a potential conflict of interest.

Copyright (C) 2019 Brunyé, Hussey, Fontes and Ward. This is an open-access article distributed under the terms of the Creative Commons Attribution License (CC BY). The use, distribution or reproduction in other forums is permitted, provided the original author(s) and the copyright owner(s) are credited and that the original publication in this journal is cited, in accordance with accepted academic practice. No use, distribution or reproduction is permitted which does not comply with these terms. 